This is the accepted manuscript of the article, which has been published in Communication and Sport. 2019. https://doi.org/10.1177/2167479519878666

Coaches’ Self-Initiated Complaints about Referees in Ice Hockey Postgame Press

Conferences

Samu Pehkonen, Tampere University

ABSTRACT: The formulation and management of coaches' dissent with referees in a setting where complaints may be sanctioned presents an empirical test for the "dilemma of stake." This article analyzes how ice hockey coaches raise complaints against referees in post-game press conferences (PGPCs). Based on the conversation analytic study of video recordings from Finnish and Swedish men's (semi-)professional ice hockey leagues, this article posits that complaints are formulated within assessment sequences and are made hearable as complaints through implicit and explicit verbal and bodily cues. The article also suggests that while referees are the non-present third-party in the PGPC, the design of the complaints provides opportunities and places obligations on the participants present in the PGPC (media representatives, game officials, and the opposing coach) to (dis)align with the complainer. Finally, the article reveals a tension between the normative expectations of the coaches to stand by their team, which may include criticizing referees, while respecting referees as guardians of sporting ethics and acknowledging the increased demands for media attention.

Keywords: complaints, conversation analysis, emotion, post-game press conference, recipient design, referees

This study focuses on (semi-)professional ice hockey coaches' formulations of complaints against referees in post-game press conferences (PGPCs). By engaging in criticism of the referees and their decisions, coaches operate in potential tension between three communication regimes in sports. First, coaches should recognize referees as the undisputed guardians of sporting ethics enforcing the rules of the game (Praschinger, Pomikal, \& Stieger, 2011): coaches should make no effort to undermine the authority of referees, nor should they question the honesty or skills of the referees (IIHF Official Rule book 2014-2018). Second, coaches are expected to behave as analytical sense makers (Goosby-Smith, 2009) for their team through their descriptions of the game, and, if necessary, they should defend their players against any perceived injustice. Third, there is an increased demand for media attention by the sports industry (Kassing et al., 2004), especially in semi-professional leagues with limited financial 
resources. For this reason, coaches are expected to provide newsworthy comments for the media to report. Thus, a “dilemma of stake” (e.g., Edwards \& Potter, 1992; Potter, 1996) arises: coaches are expected to comment on incidents that are observably a "complainable matter," but whose "complainability” makes those incidents risky for coaches to comment on.

What follows is a conversation analytic treatment of PGPCs as public occasions for coaches to draw attention to any perceived injustices caused by referees. By analyzing video recordings of PGPCs in Finland and Sweden-two major European nations in the ice hockey community-I show how this maneuvering of complaints is enacted in an interactional manner. Based on previous research, I will introduce PGPCs as a setting for communication and then focus on research on complaints and complaining as an interactional practice. My objective is thus to bring together two hitherto separate fields of study to better understand how the mediatized context of complaining affects the formulation of complaints.

\section{Sports, Media, and Refereeing}

Previous studies on pre-, post-, and in-game interviews cover various aspects and methodological approaches to the construction of sports in and through media. For example, Kurtz (2019) has discussed issues of morality within sports being brought to greater attention through an NBA press conference. Denison and Markula (2005) have analyzed Ethiopian longdistance runner Haile Gebrselassie’s press conferences as a performance, emphasizing the ways in which African distance runners are imagined by Western sports journalists. Bell and Hartman (2018), for their part, have shown how the tennis player Maria Sharapova used the "stealing thunder" crisis communication strategy (in anticipation of negative information being revealed about a person, that person chooses to reveal it first) in her press conference to defend herself against her drug suspension. Finally, Waymer and Bradler (2018) have asked whether 
professional athletes are free to express how they truly feel in their analysis of NFL player Marshawn Lynch's answers in a post-game interview after his team lost the Super Bowl. In these studies, mediatized encounters are framed as important parts of the working of the sports industry, which expects emotional content from the athletes and coaches alike.

Another line of research features on-pitch interviews and how they contribute to the production of sports culture (e.g., Smith, 2009; Rhys, 2016; Wilton, 2017). These studies have pointed out particular rules and conventions within sports communities that determine how membership (questions of belonging) is communicatively produced. Rhys (2016), for example, has pointed out that sports interviews are a particular form of interview where the typical informationseeking question-answer pair is replaced by reporters producing professional assessments of the game and the players confirming those assessments. Rhys eloquently shows how the aim of on-pitch interviews is not primarily to produce new knowledge but to conform to existing categories of interpretation expressed between competent members of the sports community.

From a methodological point of view, the above studies show the advantages of a microanalytical and members'-point-of-view approach to communication: they demonstrate how norms in a particular sports community are oriented by the members of that community, and how the members solve any problems related to following those rules. In the same vein, analyses of language use and coach talk (Llewellyn, 2003) have focused on how managers and coaches evaluate players and referees (Caldwell, 2009; File, 2017; Wilton, 2017), and how coaches manage defeat and their emotions in the presence of the media (Emmison, 1987; 1988; Gallmeier, 1987). Of special importance is Emmison's work on “defeat talk.” Based on 103 televised post-event interviews in thirteen different sports, Emmison (1988, 241-242) states that coaches tend to avoid blaming others for their team's bad performance; instead, they prefer 
formulations that aim to look and move forward from a single defeat. This holds true even when reporters suggest “controversial circumstances”-such as bad refereeing-being the cause for the loss, as the coaches are aware of the ever-present risk of showing bad sportsmanship.

Building on Emmison's findings, File (2017) has provided a sociolinguistic analysis of soccer managers' strategies to respond to interviewers' invitations to comment on controversial refereeing decisions. File shows how managers can skillfully elude invitations to provide their opinions on controversial refereeing; they neutralize the interviewers' critical assessments of the refereeing or use humor to negotiate their public image and code of conduct, but they occasionally also imply that the referees' incompetence was the reason for the outcome (File, 2017, 76). These strategies offer tacit opportunities for managers operating in a potentially dangerous interactional context where there is a threat of punitive actions and the risk of being seen as a complainer.

Finally, in her analysis of two varieties of post-match soccer interviews (PMIs), Wilton (2017) contrasts the spontaneous immediacy of on-the-pitch PMIs with the more ritualized forms of televised PGPCs, which are characterized by a rigid question-answer structure and formulaic language. As Wilton states, both formats are established parts of the full television broadcast of a football match, which may have a live and online audience potentially in the millions. The semi-professional ice hockey PGPCs analyzed here, however, are at the other end of the sports media spectrum: the immediate audience may consist of a handful of journalists, while the uploaded recording from the PGPC may attract a couple hundred online viewers. The only way to gain media attention beyond the fans of the local club may indeed be to have coaches who are regarded as linguistically imaginative and competent to produce newsworthy assessments. 
In sum, communication scholars have shown how the media constructs the ways in which the sports community talks about itself. Next, I will address complaining in more detail through studies in conversation analysis in an attempt to locate the practice in talk-in-interaction.

\section{Conversation Analytical Studies on Complaints}

This study uses conversation analysis (CA), a well-established approach to the study of both verbal and non-verbal conduct produced as a result of everyday interactions in mundane and institutional settings (e.g., Sidnell, 2010). In attempting to discover and describe interaction as the orderly thing that it is, the organization and construction of argumentative interaction has been a central topic in CA (e.g., Pomerantz, 1984; 1986; Drew \& Holt, 1988; Potter, 1996). Starting from an understanding that norms represent routine behaviors expected of people when interacting with other members of society, also complaining has been approached as a social action with normative consequences for the participants. As Antaki $(1994,74)$ notes, speakers "treat actions, behaviors, perspectives, attitudes, etc., that they allege their co-participants have performed or exhibited, as complainable.” Complaining in story-telling is a sequentially organized phenomenon where a complaint (as the first part of an adjacency pair) is followed by the recipient's preferred affiliating or dispreferred and disaffiliating response (Drew \& Walker, 2009). Participants "build turns that attribute activities to their recipients and organize their talk in ways that treat these attributed actions as shortcomings and faults, etc., by indicating that $[\ldots]$ they are considered to be unsatisfactory, undesirable, unacceptable or blameworthy" (Antaki, 1994, 74). Whether this is the case or not, speakers still face the task of representing a description of events as if their descriptions were independent of their own stake (Potter, 1996, 25). In sum, CA studies have shown in detail the members' methodic ways of entering, maintaining, and resolving disputatious sequences of talk. 
The relevance of CA for this study lies in its ability to show how coaches' complaints about referees are formulated and sequentially organized within PGPCs. Complaining can be achieved and formulated in various ways through "noticings," polar and wh- questions, warnings, assessments, and denials of rights (Monzoni, 2008; Schegloff, 2005). While these types of complainable matters occur in everyday interaction-from children playing (Goodwin, 1983) to acquaintances conversing over the telephone (Edwards, 2005)—some interactional contexts are organized around receiving and dealing with complaints, such as interactions involving the police and mediation services (Stokoe, 2009). Some are even constructed around creating and maintaining an argumentative conversational environment, such as radio talk shows or political panel discussions (Hutchby, 1992). PGPCs are particularly interesting environments for complaints, as criticizing referees is a normative violation. However, if coaches are to formulate publicly their dissatisfaction with referees, the PGPC is an ideal forum for such claims because those present have just witnessed the same game events.

An important distinction in the formulation of complaints concerns whether the target of the complaint (the complainee) is present or not when the complaint is delivered (e.g., Ruusuvuori \& Lindfors, 2009; Traverso, 2009; Ruusuvuori et al., 2019). In line with research on "third party complaints," the targets held responsible for the complainable matters of analytical interest here are referees who are not the recipients of the complaint (as they are not present in the press room to respond). Complaints about referees are directed to the multi-sited audience, which consists of both the audience present in the pressroom and the audience reached through the broadcasting service. Unlike in previous studies on complaints, it is not always straightforward to nominate even the primary recipient of the complaint. Because of the lack of a direct (dis)affiliating response from the complainees, the four-stage sequence structure of 
complaints identified by Traverso (2009) - initiation, core part, complaint development, and closing - can take place over a lengthy sequence. As a result, the complaint may be left hanging in the air, making it possible for the other coach or reporters to pick it up, or it may be upgraded with another round of accusations. If the complainer prompts an immediate response from, for example, the game official whose duty is to be present in PGPC, then the complaint must be formulated differently.

This leads to another core feature of the complaining activity in PGPCs, namely its orientation toward obtaining the affiliation of others. Complaints can be designed to include other participants in PGPCs and lead to collaboratively managed complaints (cf. Heinemann, 2009), and this should be available also in the design of the complaints. First, since game officials and referees are by default on the same side, game officials should not affiliate with the complaints delivered by coaches. The two coaches, however, can make referees a collective target for complaints, as refereeing always influences both teams. The teams may have been influenced differently, however. If the opposing team is seen to have benefitted from bad refereeing, a formulation that seeks an affiliating response from the audience is likely to be preferred. When both teams have suffered from the refereeing on an equal footing, then the complaint might

include elements that provide opportunities for alignment by the other coach. Participation in successful complaining is thus constituted through and influenced by the roles the participants play and the social activities (managing victory vs. defeat) they engage in as members of the sports community.

\section{Data and Method}

The methodological approach for collecting and analyzing the video data from PGPCs consisted of five phases. First, a decision was made to concentrate on the Mestis, the Finnish 
men's second-tier national ice hockey league and to cover initially a period of ten years (up to 2017). The reasons for selecting the second-tier league included the semi-professional nature of the league and its variety of high-visibility incidents between controversial coaches (who often are former professional players) and amateur referees. Second, official sanction records were examined in cases where coaches had received sanctions for criticizing referees in PGPCs. All reported sanctioned cases (where a video recording from the PGPC was available from the public domain from sources such as YouTube) were selected $(n=25)$. This is a small number of cases compared to around 300 games played per regular season. Third, a nonsystematic survey of another 25 videos from both Finnish and Swedish leagues was conducted to search for further cases of both sanctioned and unsanctioned complaints. The resulting 50 videos were considered a sufficient convenience sample to proceed with in a single-case analysis (see, Robinson, 2007 on “CA and numbers”). Fourth, the resulting corecollection of 31 complaint sequences was identified and transcribed following a simplified version of CA transcription conventions. Fifth, the collected cases were analyzed using CA, which proceeded by examining how coaches designed - turn by turn, sequence by sequence (e.g., Sidnell, 2010) — their dissent with the referees to answer the following research questions:

Research question 1: How do coaches initiate and manage (both verbally and non-verbally) complaints in a context where complaining is sanctioned?

Research question 2: How does the turn design and formulation of complaints build on the other participants’ obligations to (dis)align with a complaint?

The analysis and results are presented in the form of four types of complaint formulations: implicit complaints and three types of explicit complaints. The presentation is not compatible 
with detailed quantitative analysis — that is, all four categories of complaints could be used within a single press conference. As Robinson $(2007,73)$ states, "some practices are more common than others, and their frequency does not necessarily indicate their importance.” However, all implicit complaint sequences $(n=6)$ were found among non-sanctioned cases, while the three categories of explicit complaints where more or less equally prevalent. Finally, while the formulations of the complaint do not necessarily differ grammatically across these categories, the normative and interactional implications do differ.

\section{Analysis}

\section{Implicit Complaints}

The first category of complaints occurs within the ongoing project of delivering a chronological description of the game. In Excerpt 1, the coach of the visiting team has produced his chronological assessment of the game. The chair nominates Peter (the home-team coach) as the next speaker. Peter starts his description of the game event with a positive statement about his team's performance early in the game.

Excerpt 1. Örebro HK-Almtuna IS 0-2 December 21, 2012 (Swedish) $^{1}$

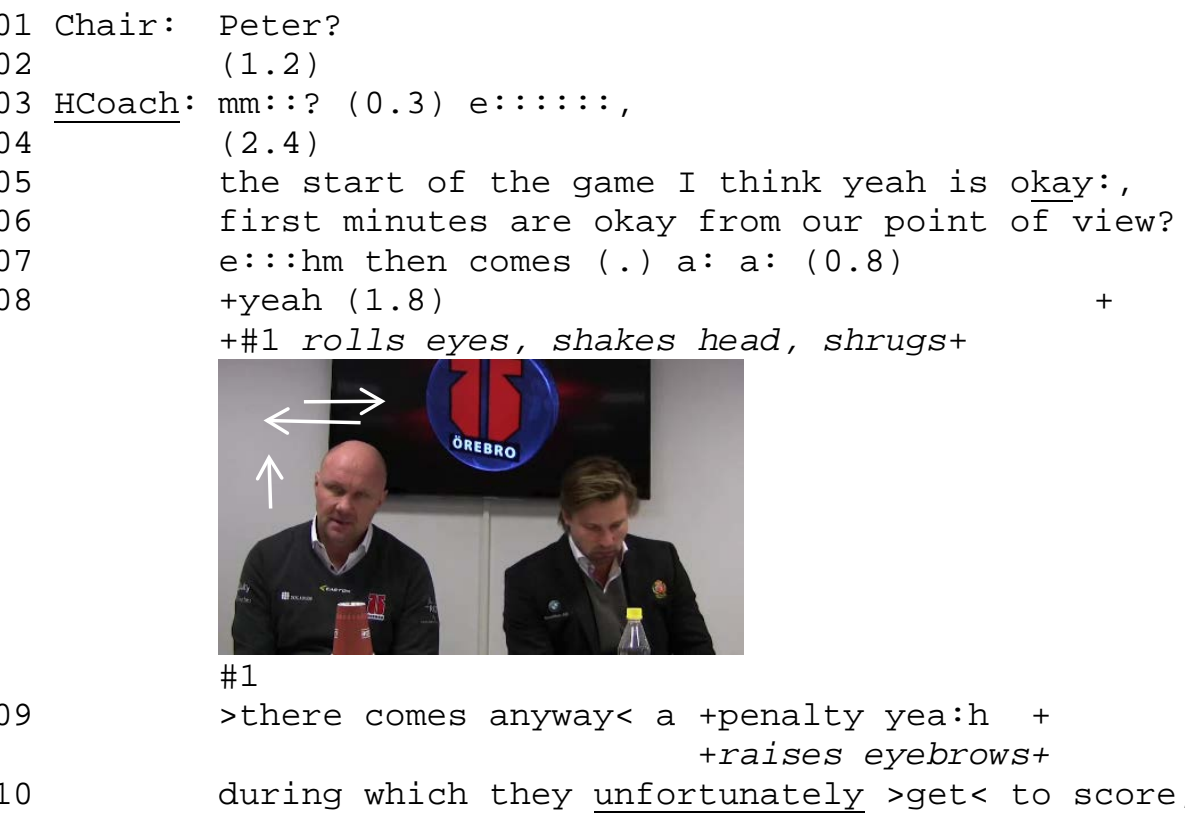




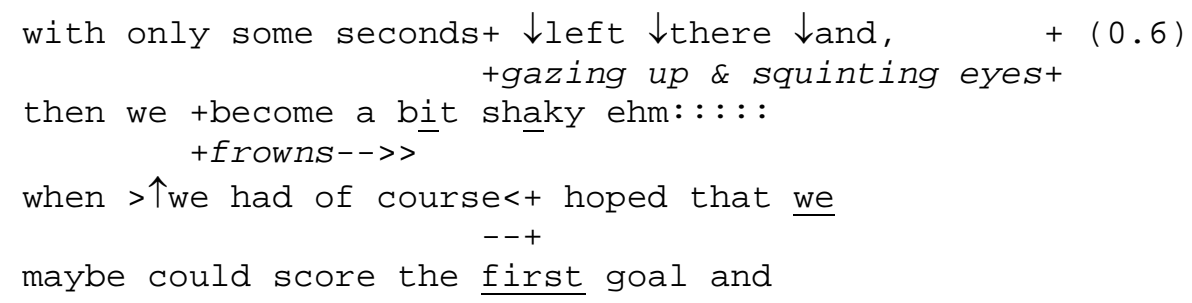

The complaint here is implicit in the sense that it is not designed to be heard as a complaint; yet we hear it as one. The fact that it takes a while for the coach to start his turn (hesitation fillers and pauses in lines 2-4) is the first observable sign of a possible ambivalence in terms of what type of description he should produce (see Jefferson, 1974; Ruusuvuori et al., 2019). The complainable matter, a penalty, is presented indirectly, as embedded within the syntactic or grammatical unit of turn formulation (lines 7-9). The narrative unit, “then comes a penalty and they manage to score,” would be complete even without the hesitations ( $a$ : a: yeah anyway a penalty). In addition, his facial expressions, raising his eyebrows while searching for a word ${ }^{2}$ (Goodwin \& Goodwin, 1986) and a head tilt followed by a shoulder shrug (Debras, 2017), mark the talk as separate from the overall narrative, which he delivers with minimal gestures and a static positioning of his body (cf. Peräkylä \& Ruusuvuori, 2012). The perceived injustice is further emphasized by the penalty resulting in the opponent "unfortunately" scoring just before the penalty ended. All these features mark the turn as an indirect complaint with no nominated complainee. Typical of implicit complaints, the narrative sequence of the assessment of the game continues, and the coach does not return to the complaint.

In Excerpt 2, the coach of the visiting team is finishing his game assessment. He gives credit to the players who continued playing in the third period, even though they were losing by several goals.

Excerpt 2. Hokki-Kiekko-Vantaa 6-3 February 7, 2014 (Finnish)

01 VCoach: we pull out the goalie and $(0.7)$ we don't manage to score so 
07

08

(.) there's simply (0.4) nothing at all we can do about it, but a $\uparrow$ good fight from the team, $(0 . \overline{7)}$ from the team and (.) those who $(0.5)$ Tour fans who watched this game via (.) ((streaming + service $))^{-}$so, $+(0.5)+$ I simply just can't (.) +raises eyebrows+ +...-.-head shakes-..... turn into+ a whistle.=

Instead of stopping after finishing the turn construction unit (TCU) "but a good fight from the team" in line 3, the coach repeats the phrasal TCU "from the team" and extends his assessment by taking the outsider's point of view. He refers to the interpretation that fans might have gained from following the streamed game. In lines 5 and 6, he produces the complaint unit "I simply just can’t turn into a whistle.” The complaint is formulated by contrasting the roles of the coaches and referees that are, in reality, separate and non-interchangeable. With this notification, the coach emphasizes the things he cannot influence, while simultaneously implicitly indicating another group of participants should indeed have blown the whistle: the referees. The complaint is formulated with the Finnish clitic particle -hän (roughly translated here as "simply"), which is generally used in disaligning responses to show the obvious state of affairs. Also noteworthy are his facial expression (raising his eyebrows) just before launching the complaint and his head shaking while producing the complaint unit. He then returns to his role as a coach who, according to the rules of fair play, should not comment on or assess the actions of referees (line 7). The sequence here serves the double role of showing the coach and his team having done all that was in their power, while at the same time referring to the referees having denied them the help they would have deserved in the form of penalties awarded against the opponent.

A common feature in implicit complaints is that while the complaint units are often subtle, they are sequentially produced by using various interactional methods to prepare the ground for making such units hearable as complaints. As in excerpts 1 and 2, pauses, hesitations, or 
affective facial expressions or gestures mark the problem. The complaint unit in itself may be produced through the extension or circulation of TCUs from the main narrative. Implicit complaints are often used by coaches as a way of managing defeat in a non-grudging way.

\section{Explicit and Affiliation-Seeking Complaints}

Explicit complaints are designed to invite accounts from the parties held responsible for the complainable matter and/or to seek the co-participants' affiliation for the speaker's stance. To succeed in this, the speakers balance between formulating the complainable matter, nominating the complainees held responsible, and providing legitimate reasons for their complaint. In other words, while engaging in complaining, coaches avoid being seen by others as complaining about non-complainable matters. For this reason, complaining sequences in face-to-face interaction consist of the slow progression of the complainable matter to the expression of affiliation by the recipient (Traverso, 2009). This is particularly true in PGPCs, where turns are pre-ordered in the sense that the current speaker can hold the floor and build up the justification for the complainable matter without the risk of being interrupted. The next excerpt (3) is a lengthy one, as there are several observable signs in the preceding turns that prepare the ground for the complaint.

Excerpt 3. Färjestad BK-HV71 3-5 March 17, 2012 (Swedish)

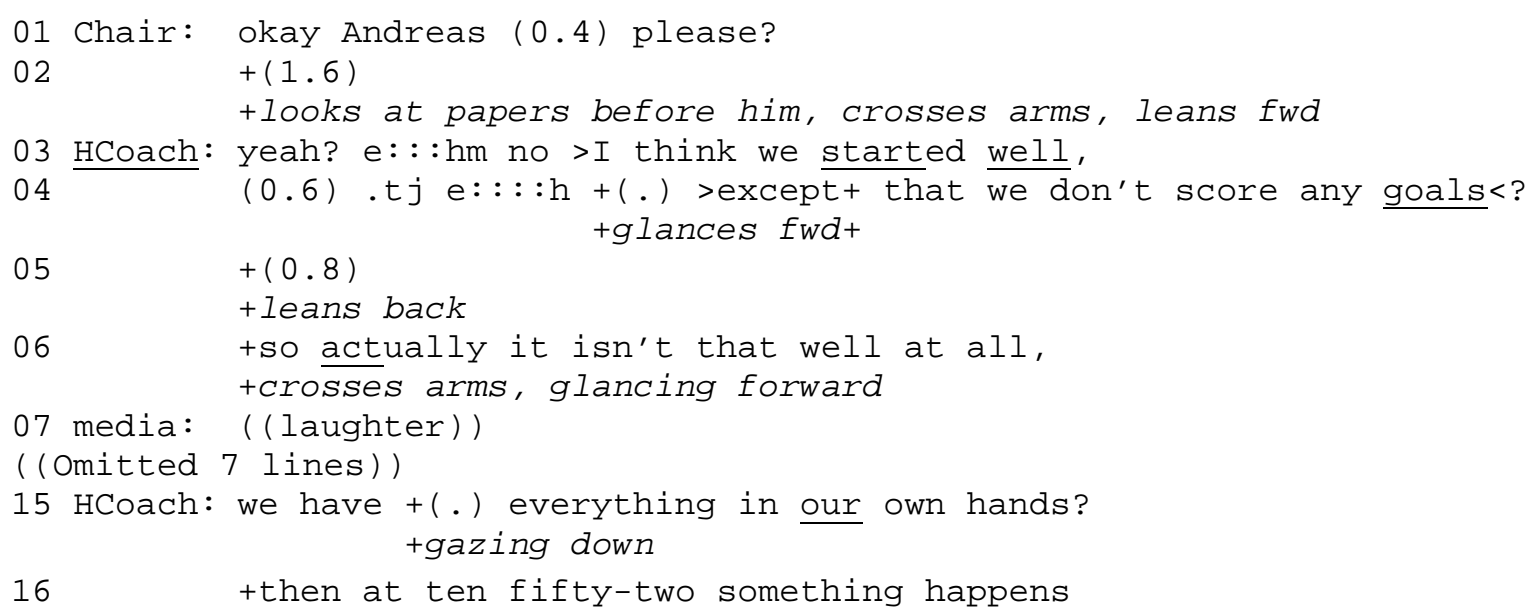



+-----finger up-.--+ +-pointing->>

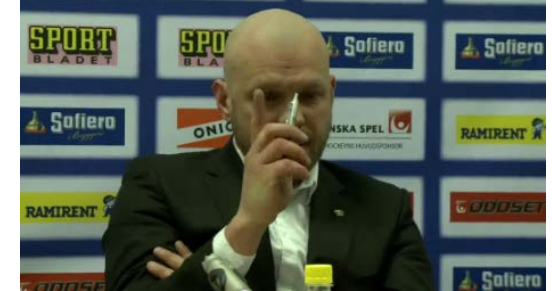

$>$ we haven't whined< about referees+ anything but +

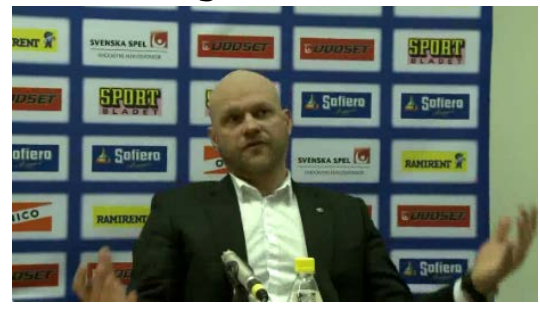

$\# 3.2$

+get a minor only (.)

+leans back, crosses arms on chest+

$+\# 3.3$ and (.) + and they score four three.

+glances right+

$$
\text { (1.1) }
$$

+ nods right $++k n i t$ brows +

$+(1.1)$

+looking at papers on table-->>

=then it's good night. + glances up-->>

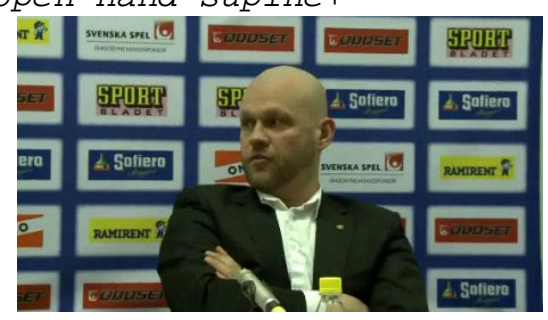

\#3. 3

+if you know+ +any thockey so >you understand<

that it >should have been< a >double penalty< there.

and $\uparrow$ then (1.6) Jason +Krog scores five three.=

The coach of the defeated home team presents his description of the game in chronological order, beginning with the start of the game, using cynical assessments (lines 3-6) for successful audience affiliation (line 7), showing his powerlessness against the bad refereeing (especially the shrug in image 3.2; see Debras, 2017), and ending with a laconic "good night" statement (line 33) that resulted from the decisive goal scored by the opponents. While the coach is 
showing his disappointment with the final score (and implicitly with his team performance in the second period in the omitted lines), these displays of his stance are also produced to manifest a particular event within the game. From the line 15 onwards, by referring to the precise moment of 10.52 in the middle of the third period, he produces the complainable matter, a misjudged penalty call.

The weight of this call is accounted for in four ways through the composition of the complaint. First, he mentions twice the precise moment of the unjust refereeing decision with an accurate and embodied description of the event and its participants. Second, the description also has a moral element, as he aligns with the penalty given to his player but refuses to accept that an opposing player got away with a similar (if not an even worse, vengeful) offense. Third, by glancing quickly toward the other coach when mentioning the opponent's power play goal (line 26), he points out that the other coach benefitted from the bad refereeing. Finally, the last resource used here builds on “stake inoculation” (Potter, 1996, 25)—a counter-dispositional practice of the unbiased expert view — when the coach claims that "anyone who understands anything about ice hockey” would make the same interpretation (line 29). In sum, the complaint against the referees is designed to be alignable for the audience and against the other coach, whose team is portrayed as having benefitted from unjust refereeing.

It is important to note how coaches formulate complaining as a relevant and justified activity in this particular case. In Excerpt 3, the coach produces a pre-emptive warrant for his complaint-to-be by stating that he has not whined about referee calls (lines 15-16). "Not being a whiner” is one of the typical warrants coaches use to point out that they only question referee calls that have indeed been objectively unjust. We can contrast this with Excerpt 4, which 
features a similar entry into the complaint sequence, but one resulting in the opposing coach producing affiliative laughter in response.

Excerpt 4. TUTO-HCK 2-1 GWS February 5, 2014 (Finnish); sanction: two games missed and a fine of 1,000 euros

01 HCoach: forty-eight and a half games I've abstained from whining about

02

03

04

05

06

07

08

09

10

11

12

13

14

15

16

17

18

19

20

21

22

23

24

25

26

27

28

29

30

31

32

33

34

35

36

37

38

39

40

41 HCoach:

42

43 VCoach:

((Omitted 12 lines))

56 HCoach: I lost my appetite for this ( $\odot .5)$ game as it comes to those 57

58

the refs so that I don't shout to the rink and don't (.)

discuss $(0.7)$ with them during intermissions,

but now it just has turned into so that the more (0.7) their

$(0.7)$ crews shout and scream the more it always turns (.) to their benefit and, $(\odot .8)$ we probably should've been given three four five penalties more.=and then on the top of that today was $(0.6)$ <the funniest> I've <ever> seen in any ball game culture.= we play to win (.) the regular season (1.0) a one goal game (.) so you give there in the mid third period $\bar{a} \uparrow l$ inesman comes

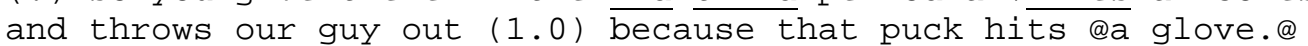
$(0.8)$

there is next to the board (.) that kind of battle $\uparrow$ fifty fifty and it <the puck bounces $\uparrow$ from the bench and hits the glove, > (๑.9) 个and $\uparrow$ that $\uparrow$ chimpanzee throws us a minor penalty. $(1.3)$

so (.) @that for real@we- (. )

we are working hard here year after year (0.4)

we practice two times in the morning in the evening so, (1.1)

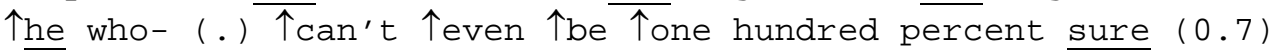

if that <@glove@was inside the rink> (0.5) or something.

if the rules say.=you can't <for god's sake> (0.7)

give $(1.0) £ t(h) h a t$ type of penalty in that type of situation. $£$ $(0.8)$

个you just can't.

$(1.0)$

it's like <raping> all conformity with the laws of a ball game. $(0.6)$ so that you then $\overline{\text { eagerly }}(1.1) @$ tiptoe to the head ref

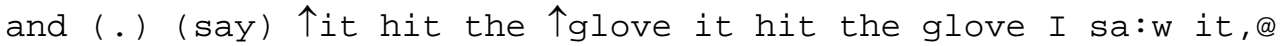
$(0.7)$ <oh my god>. ( 0.7$)$ in an adult game.=

we are playing for really high stakes here.

$(0.8)$

you've got to be hundred percent sure.

$(1.6)$

>so so< (.) 个now (.) 个now it feels like kind of bad,

(1.1) that three points were there first available and then

all of a sudden they were not $(0.6)$

this is really getting under my skin so that $(0.5)$

+ I really $£ g o$ out of control. $£$

+ glances at vcoach

( (laughing) $)(1.6)$

EI mean went really out of control.£ (.)

[such bad feelings] I got from that game.

(1.1) guys with striped shirts.

$(1.1)$ 
Explicit complaints make emotional investment relevant and at least potentially acceptable by the potential recipients. After the pre-emptive warrant (“I haven't whined..." lines 1-3), the sequence is followed by a claim about other coaches resorting to influencing referees (lines 46), an ironic statement about its effectiveness in this game (lines 6-7), and finally the introduction of a locally justified complainable matter (lines 7-15): the actions of referees in the game. The TCU starting in line 7 specifies the complainable matter, a penalty given in the third period, by using an extreme case formulation (Pomerantz, 1986): “on the top of that, the funniest, and in any ball game culture” indicates the penalty in question was observably exceptional. The chronological order of the incident is described in detail and the suprasegmental elements (smiling voice, intonation) in the production of the description mark the speaker's position that the penalty was ridiculous. In line 15, the linesman as a complainee is called a pejorative name, “a chimpanzee.”

The initial complaint is followed by a pause of 1.3 seconds (line 16), marking what would normally indicate a transition-relevant place (TRP) where a change of speaker might occur. Because of the specific turn-taking system in PGPCs, however, other participants are not expected to take a turn and the current speaker can choose to continue. This is what happens, and the coach produces an extension of his complaint (lines 17-25). The complaint is upgraded in terms of the selection of words (the use of profane language, line 22), the style of talk (imitation, stress; especially in lines 28-29 produced in a high-pitched, childish manner), and the explication of the unjust nature and uncertain grounds for giving the penalty. He also contrasts the work done by the players to be able to play and the (supposedly) unskilled referees who are unsure of how to apply the rules. 
Again, the conclusion-type TCU “you just can't,” followed by the one-second pause in line 26, offers a space for the coach to end the complaint sequence and proceed with the assessment of the game. What happens instead is a second round of complaint extension, resulting in a further upgrade in terms of vocabulary, idioms, and justification for the consequences of such misjudged penalties. Finally, after the next TRP (in line 33), there is progression in the coach's description, as he verbalizes his emotional stance, thus providing himself an interactional way out of the complaint. ${ }^{3}$ At this point (in line 39), he looks for the first time at the other coach sitting next to him. The other coach responds with laughter, a highly affiliative response and a face-saving act for both parties. One can argue that while the coach initiating the complaint might recognize his normative violation of the rules of conduct in PGPCs ("ugh I have spoken” in line 59 affirms him having expressed his genuine opinion while simultaneously closing the sequence), he has nevertheless managed to recruit the other relevant participant in the production of the justifiability of his complaint. By doing this, the complainability - even if unjustified - is collaboratively produced. It becomes something that coaches are legitimately able to do, even if sanctions are inevitable.

\section{Explicit Complaints in Search of an Accountable Recipient}

Efforts to recruit the other coach into the complaint are risky, however, as the other coach may not align with the complaint (see Drew \& Walker, 2009). If the other coach refuses to align with the criticism toward the referees, then he is also likely to be treated as someone not respecting the game. Although the initial complaint is aimed at the referees, often the complaint is made also because the other team gained an advantage from those unjust calls. An example of this is provided in Excerpt 5. 
Excerpt 5. Hokki-LEKI 4-3 OT December 20, 2013 (Finnish)

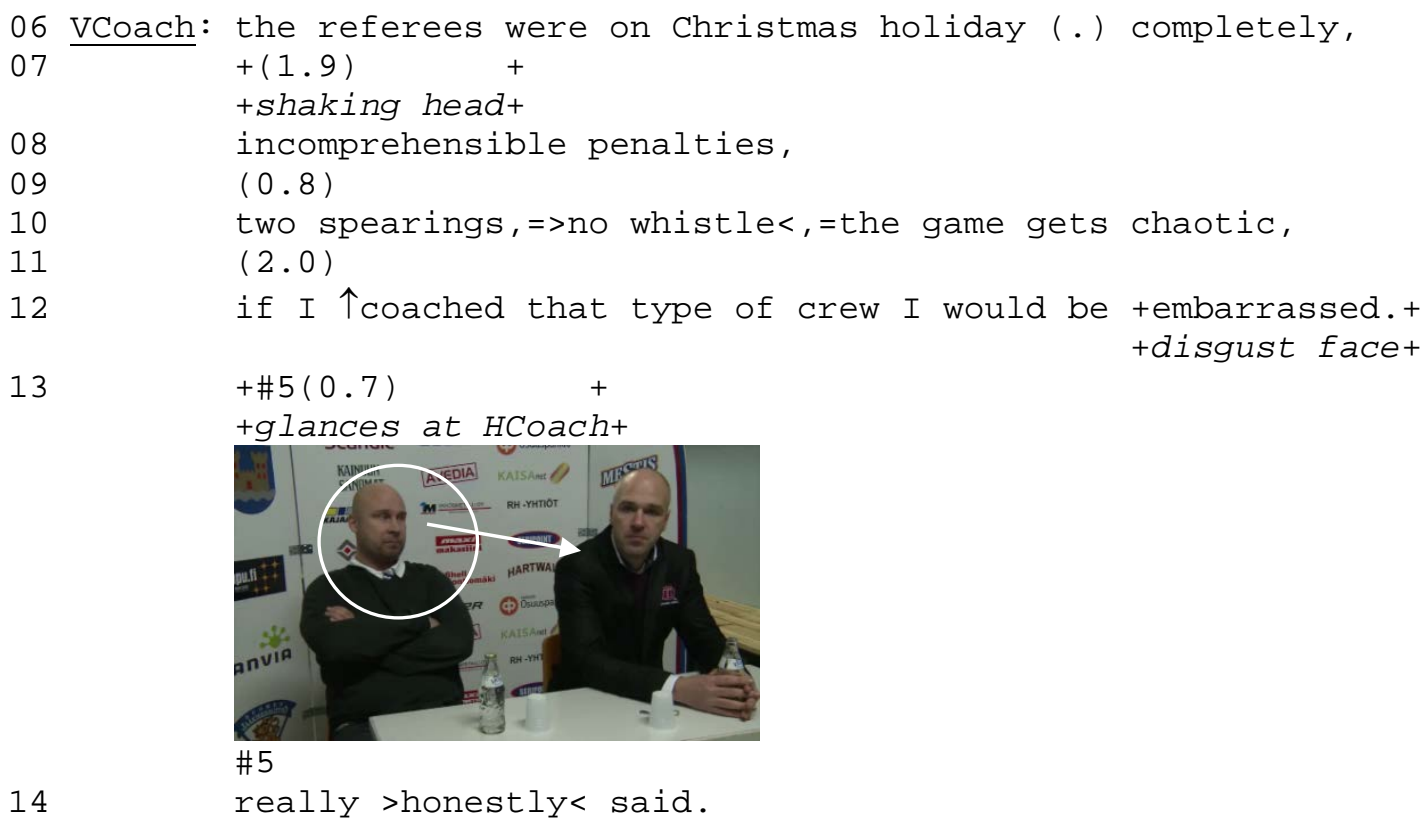

While the TCU "the referees were on Christmas holiday" may be a reference to the game being played just before the Christmas break, it is heard as a complaint in terms of the referees not doing their job properly, but instead giving “incomprehensible penalties.” The reference to "spearing” (poking an opponent with the tip of the hockey stick's blade, which should automatically result in a major penalty and game misconduct) includes the two parties held responsible: the referees who failed to judge those incidents properly and the players from the opposing team who committed those offenses in the first place. The coach of the visiting team then expands the complaint to include the home-team coach, who he sees as being partly responsible for not controlling his players. Glancing at the home-team coach and producing the TCU “I would be embarrassed,” the away-team coach makes his opposing counterpart partly accountable for the refereeing. Complaints that make a participant present in the PGPC accountable are not necessarily followed by a direct response, because the fixed structure makes it easy for the recipient to evade the complaint. ${ }^{4}$ Knowing that the other coach is unlikely to provide a response to his accusations, the coach leaves the room. 
Cases like this belong to the category of explicit complaints, where the complaint is often emotionally allocated to particular recipients, who are held responsible for providing accounts of the complainable matter. If these recipients are present, they can be made accountable for producing a response to the complaints through questions directed to the recipients. The inability or unwillingness to provide such a response produces further accusations. Emotionally laden complaints seem often uncontrolled and impulsive. They are also the complaints that attract the most media attention - and sanctions.

One particular case involves a referee and a game observer, the latter of whom was indeed present in the PGPC as representatives of the game officials. Excerpt 6 provides an extreme example of a complaint targeted at a game observer. Preceding the start of the excerpt, the coach of the visiting team has turned down the chair's invitation to comment on the game, and instead has introduced issues (Excerpt 6a) he wants to discuss with the game observer, whose duty is to report any notices and shortcomings during the game event. The problem is that the observer is currently unavailable. When he finally enters the room, the coach repeats his question (Extract 6b). For the sake of brevity, I have omitted those lines in the transcript that include complaint formulations already discussed (such as the circulation and extension of complaints) in connection to the previous excerpts.

Excerpt 6a. Hokki-Kiekko-Vantaa 3-2 GWS October 9, 2009 (Finnish); sanctions: two games missed and a fine of 1,000 euros

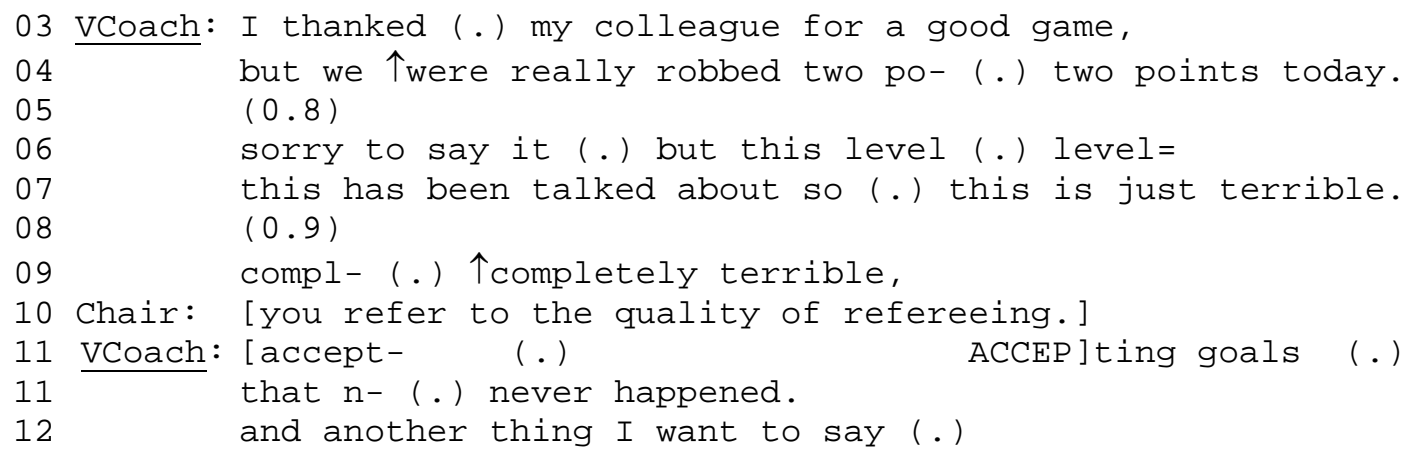


a game misconduct for our captain, thei- their one player comes to take away= challenges (.) @shall you@ (๑.5) and we get the game misconduct. (1.7)

SO MUCH understanding these boys have for this game.

(1.1)

they make clowns of themselves and these same clowns are every time here $(0.5)$ when we are here.

$(0.8)$

+this wasn't for you.

tturns facing the other coach

The coach opens the sequence with a complimentary turn, thanking the other coach for a good game. By using the past tense ("I thanked”), however, he distinguishes between what he has already done behind the scenes (i.e., shown respect to the opponent) and how he intends to use the rest of the public PGPC. He immediately continues his turn with the complainable matter, “but we were robbed two points today” (line 4), which presents his team as the victim through the selection of words ("being robbed” vs. we lost). This position of victimhood in itself would already provide a justification for his complaint, but the coach further emphasizes his right to criticize by claiming he feels regret ("sorry to say but," line 6) for having to once again take part in an ongoing discussion on refereeing (“this has been talked about so,” line 7). He then provides two examples of the “completely terrible” level of refereeing: accepting controversial goals and awarding penalties contrary to the rules. After a short description of the penalties given for a fight, he asserts that the referees are making themselves look stupid in the eyes of the players. Thus, by referring to the membership of the sports community, the coach can be seen as speaking for everyone; this is not his personal complaint (note also how he refers to the other coach as not being part of those who are being complained about in line 22).

The start of the sequence summarizes the main components of the explicit complaints: the recognizable prelude to the complaint, the use of extreme formulations (“total,” “completely”), the expressions of an emotional stance, the detailed description of concrete incidents (who did what and in which order), and the naming of those complained about (referees as clowns). The 
exceptional dimension here is that the coach insists on posing a question to the person representing the referees, the game observer.

Excerpt 6b.

((Omitted 41 lines during which the game observer is said to be on his way; vCoach refuses chair's offer to assess the game in more general terms)) 64 Chair: [okay so here was (.) something for you.]

65 VCoach: [okay so I I (.) I want to pose ] a question now.

66 thei- their player (1.1) asks ou- our captain

68 their third line forward asks our most important

69 defenseman @wanna fight (.) shall we start?@

$70 \quad($.$) and we get the game penalty they don't.$ $(1.8)$

71 Goff: yeah my duty is not to give sanctions and that stuff here but

72 well $(0.4)$ and I (.) don't comment on that here=

73 个I'm 个just (.) or I'm here just to check out that decent

$74 \quad$ language is used.

$75 \quad(2.5)$

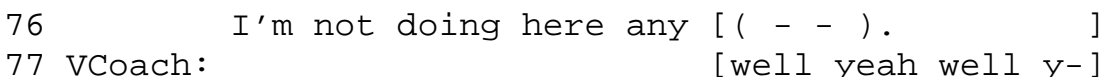

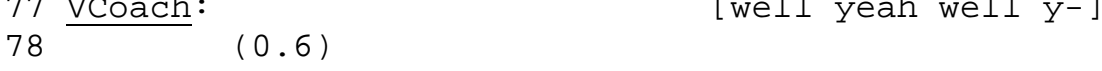

79 you can- you can for my part leave [your - ) ]

80 Goff: $\quad\left[\left({ }^{-}--^{-}\right)\right]$

81 vCoach: just (.) just (.) it makes no sense to discuss these things.

$82 \longrightarrow(0.8)$

83 +this was this was a total fucking farce this thing.

+folds a sheet of paper on the table and puts it in his pocket

$84 \quad(0.5)$

85 Goff: [ $(--)$ ]

86 VCoach: [个a total farce.]

87 GOff: decent language needs to be used.

$88 \quad(1.0)$

89 VCoach: gimm- gimme gimme a penalty gimme fines for fuck's sake.

90 (1.0)

91 fucki- (.) you come smiling here (.) smiling really

$92 @ @ d e c e n t$ language.@

When the game observer finally arrives, the coach provides a similar description of the incident of the two players starting to fight, which then led to the player who initiated the fight being given a game penalty. The observer treats the coach's description (lines 65-70) as a question (although not formulated in question form), but he declines to provide an answer. Instead, he refers to his role as an observer who does not make decisions and is not accountable for providing such an assessment. The coach, at this point seemingly frustrated at not getting the affiliating response he wants, then announces the unhelpfulness of the game observer and 
continues to describe the refereeing as a farce (lines 83 and 86). When the observer repeats that his duty is only to make sure that decent language is used and, by repeating this, suggests that the coach is not obeying the rules, the coach responds with the "gimme a penalty" turn (line 89). With this turn, the coach makes it clear he has just crossed the line where sanctions are inevitable and, therefore, he can continue his complaint sequence.

Because the game observer has become the target of the complaint, the sequence also illustrates the different components of complaints when a present or non-present party is the cause of the complainable matter. The present complainee's turns fail to produce an adequate aligning response, which can be used as a reason for further complaints. Some of the coach's TCUs address the game observer directly through his (apparently inappropriate) actions: by smiling and asking for the use of decent language, the observer is demonstrably dismissing the legitimacy of the coach's complainable matter. By repeating and imitating the observer's actions (line 91-92), the coach can, in turn, provide further evidence for the unjust treatment. The sequence continues for a while before the coach finally walks out from the room. Explicit complaints addressing a recipient present in the PGPC are likely to breach the expected structure of the institutional setting.

\section{Explicit Complaints Targeted at the Broader Audience and for the Sake of the Sport}

The final category of explicit complaints includes sequences that, while pointing out discontent with the referees in the particular game, frame the complaint simultaneously as a comment on refereeing in general. The emphasis here is especially on the disaffiliating facial expression of the other coach and the complainer's discrete exit from the complaining sequence.

Excerpt 7. Kiekko-Vantaa-Jyp-A 1-4 November 14, 2014 (Finnish)

01 HCoach: we keep on working. (.) it doesn't help to cry here 


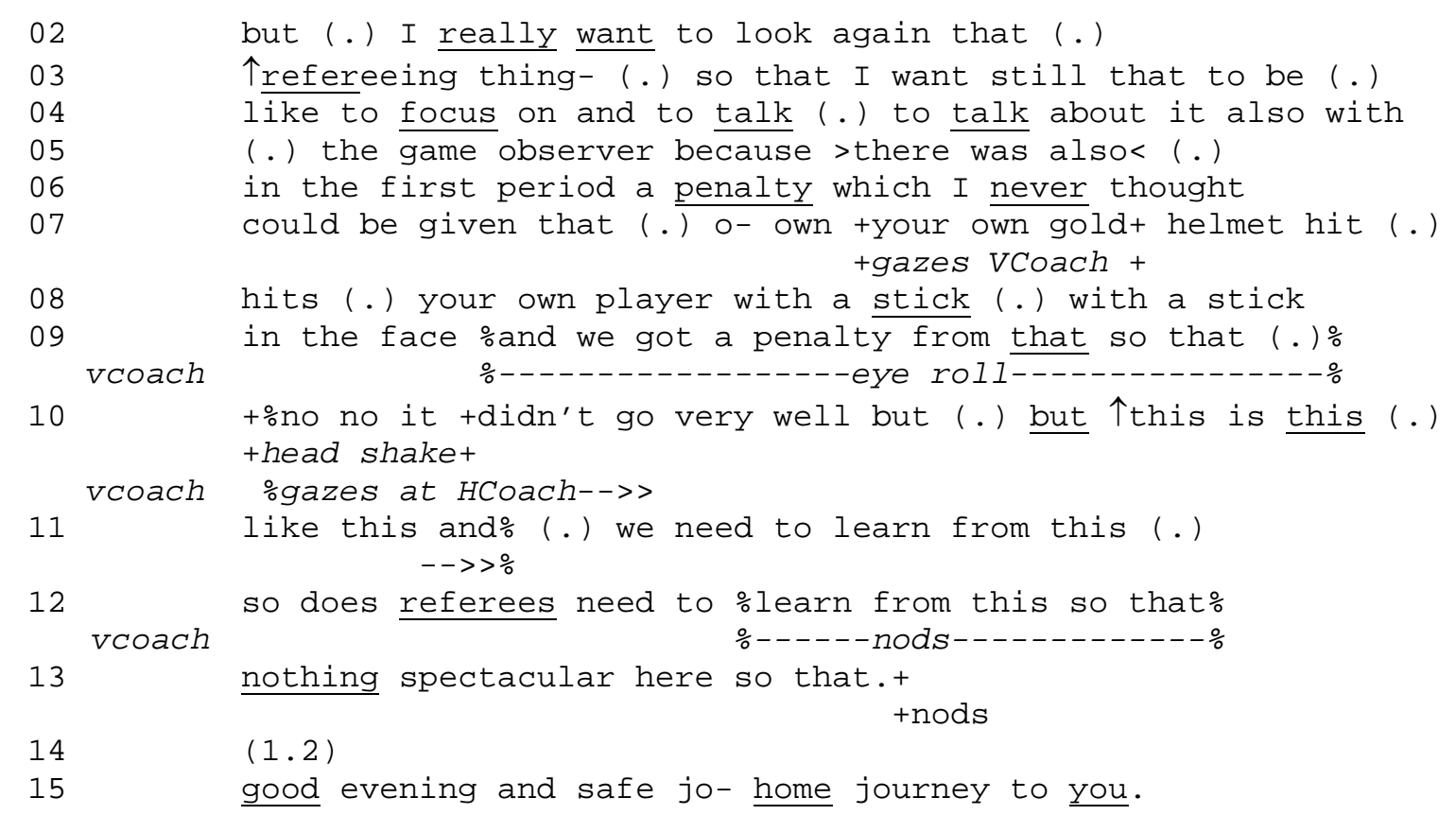

Near the end of his game assessment, the coach for the home team points out his willingness to discuss the grounds for a penalty with a game observer. He provides himself as a first-hand witness to the controversial call, stating that his player was not even part of the high-sticking. By reference to “your own gold helmet” (line 7), he addresses the other coach and a particular player on this team as participants in the contestable case. Visitor's coach immediately gazes at the speaker and then produces an eye roll, a facial expression conveying disaffiliation. Having expressed this view on the referees and having received a disagreement from the other coach, the home-team coach nevertheless provides a solid way out of the complaint: rather than walking out, he states that calls of this nature are part of the game and can never be completely avoided. He turns the complaint into a learning opportunity - for himself and his players but also for the referees. He thus eloquently combines criticism with an important element in defeat talk, namely a future-looking optimistic agenda (Emmison, 1988). In terms of turn allocation, he also very effectively uses his role as a home-team coach to close not just his turn but also the PGPC by wishing the visiting team a safe journey home. 
PGPCs have a link to the particular game just completed, yet the turns produced by the coaches also extend to the forthcoming games. For coaches who wish to discuss refereeing in general, PGPCs are one of the few public opportunities to raise their opinions on and experiences of refereeing. Rather than uncontrolled emotional bursts, it is possible to design some entries to and exits from complaint sequences, including staged performances with the coach looking at and speaking intensively to the camera. Complaining is a way of showing awareness of the normative and moral expectations, not of engaging in criticism of referees per se.

\section{Discussion}

This article used a collection of complaint sequences in Finnish ice hockey post-game press conferences, and like similar studies (e.g., Mellick, Fleming, \& Davies, 2007; Kassing, \& Anderson, 2014; Cranmer, \& Buckner, 2017), it focused on interpersonal communication and dissent in sports. Typically, studies on dissent are based on interviews or other researchercontrolled methodological protocols that aim to uncover what participants make of their communication practices. However, the methodology followed here-i.e., conversation analysis-studies the coaches' own orientation to their reasons and basis for criticizing referees. This orientation is analyzable through video recordings as sequentially organized, actual, and occasioned practices performed in the course of talk-in-interaction (Pomerantz, 1984). This enables us to focus empirically on what coaches actually do rather than what they think they do or what we think they think when they engage in criticizing referees.

I have shown how ice hockey coaches in post-game press conferences self-initiate and publicly handle controversial issues in refereeing while simultaneously trying to prevent being seen as someone who "always whines.” As a part of their descriptive assessment of the game, coaches can “do complaining,” point out the “complainable matters," and nominate complainees either 
implicitly or explicitly by using various linguistic (hesitation markers, extreme case formulations) and multimodal resources (facial expressions, gestures, and gaze). I have also shown how turn formulation and turn design, suprasegmental elements in talk, and facial expressions and the bodily delivery of assessments are crucial in enacting complaints for affiliation-seeking purposes and formulating stakes by providing pre-emptive warrants to protect their accounts. If the coach initiating the complaint fails to receive an affiliating response from the other participants (most importantly from the other coach), the likelihood increases that the initial complaint sequence extends and leads to further complaints.

Managing defeat caused by controversial refereeing can involve criticism toward referees. The difference between mocking the referees and constructively criticizing the consistency of the refereeing is not clear-cut, however. It is safe to say that implicit complaints are not necessarily even recognized as complaints by the participants present in PGPCs. All three categories of explicit complaints analyzed here include both sanctioned and unsanctioned cases. Based on the sequences analyzed, it is difficult to see why the Discipline Committee sanction some cases while other cases remain unsanctioned. The Committee admits that they "fully recognize that during the game emotions get heated and occasionally things are said [...] that should not be said," and that a "matter-of-fact criticism toward refereeing in general is allowed and acceptable." At the same time, however, the Committee holds that "the criticism should be directed at those concerned, instead of publicly condemning the system or decision-makers on a general level.”

This argument dismisses the fact that PGPCs are a natural platform for coaches to present their viewpoints publicly and to comment on the development of the game's culture. If discussions concerning the refereeing are held behind closed doors, players, coaches, and fans alike may 
feel they lack full membership of the community. In fact, Sanderson (2009) has shown how an NBA franchise owner's criticism of officiating expressed through blog entries promoted change within the league. This was partly because the owner's "dissent[ing] but humorous" expressions were confirmed by blog readers, creating public pressure. Similar conclusions could be drawn here: using humor and speaking on behalf of the fans or "for the game" are likely to draw attention to and create alignment with the topic rather than shift the focus onto the provider of criticism.

One limitation of this study is my specific focus on the initiation and progression of complaint sequences in the institutionalized parts of PGPCs; I have dealt with the responses by the other participants only in passing. After these initial turns, participants may engage in sequences where immediate responses are provided and further complaint sequences with nominated complainees emerge. It remains open if those sequences, including direct invitations by media representatives to criticize referees, are organized differently. It would also be interesting to expand the study to include sports other than ice hockey, and to bring in empirical evidence from the inclusion of video assistant referees (VAR). The current study already demonstrates, however, that the interactional structure of PGPCs with their fixed turn-taking system should be acknowledged as an important resource for launching and managing complaints.

\section{Notes}

1. All excerpts are presented here in translation. The titles include the following information: the names of the two teams, with the home team mentioned first; the final score (OT=over time, GWS=penalty shootout); the date of the game; the language of the press conference; and eventual disciplinary sanctions, where applicable. The current speaker and his team are underlined. Screen shots of the key gestures and facial 
expressions are provided when the quality of the original video file permits reproduction.

2. From a grammar perspective, the word searched for might be an adjective describing the penalty as stupid, unfortunate, or unjust. While the precise word remains to be formulated, the turn completion "anyway a penalty yeah" suggests that what is left unsaid is the unjust nature of the penalty.

3. In explicit complaints of this type, it is common for the coaches themselves to verbalize their emotions, such as "being pissed off" or having things "really getting under my skin.”

4. Although not the topic of this article, it should be mentioned that the media representatives play a key role with their follow-up questions. After the initial turns by the coaches, journalists can refer to the assessments, descriptions, and complaints put forward by one of the coaches and ask the other coach to comment on them.

\section{Transcription symbols}

$\begin{array}{ll}\text { underlining } & \text { - strong emphasis } \\ \text { SO MUCH } & - \text { shouting } \\ \text { - } & - \text { truncation } \\ \text { [ ] } & - \text { overlaps } \\ \text { = } & - \text { latching of turns } \\ \text { (0.5) } & - \text { pause length in tenths of a second } \\ \text { (.) } & - \text { micropause } \\ \text { : } & \text { - lengthening of a sound } \\ £ & \text { - laughing voice } \\ \text { @ } & \text { - voice quality }\end{array}$




$\begin{array}{ll}\begin{array}{l}>\text { huh }< \\ <\text { huh }>\end{array} & - \text { compressed or rush talk } \\ \cdot & - \text { slowed talk } \\ ? & - \text { pitch fall at the end of an intonation unit } \\ , & - \text { pitch rise at the end of an intonation unit } \\ \downarrow & - \text { level pitch at the end of an intonation unit } \\ \uparrow & - \text { mid-turn rise in pitch } \\ (--) & - \text { unhearable word(s) } \\ +\% & - \text { timing of embodied action } \\ \# 3.2 & - \text { the moment of the screen shot }\end{array}$

\section{References}

Bell, T. R., \& Hartman, K. L. (2018). Stealing thunder through social media: The framing of Maria Sharapova’s drug suspension. International Journal of Sport Communication, 11, 369-388.

Caldwell, D. (2009). "Working your words": Appraisal in the AFL post-match interview. Australian Review of Applied Linguistics, 32, 13.1-13.17.

Cranmer, G. A., \& Buckner, M. M. (2017). High school athletes' relationships with head coaches and team-mates as predictors of their expressions of upward and lateral dissent. Communication Journal, 74, 390-405.

Denison, J., \& Markula, P. (2005). The press conference as a performance: Representing Haile Gebrselassie. Sociology of Sport Journal, 22, 310-334.

Debras, C. (2017). The shrug. Forms and meanings of a compound enactment. Gesture, 16, 134. 
Drew, P., \& Holt, E. (1988). Complainable matters: the use of idiomatic expressions in making complaints. Social Problems, 35, 398-417.

Drew, P., \& Walker, T. (2009). Going too far: complaining, escalating and disaffiliation. Journal of Pragmatics, 41, 2400-2414.

Edwards, D., \& Potter, J. (1992). Discursive psychology. London: Sage.

Edwards, D. (2005). Moaning, whinging and laughing: the subjective side of complaints. Discourse Studies, 7(1), 5-29.

Emmison, M. (1987). Victors and vanquished: The social organization of ceremonial congratulations and commiserations. Language and Communication, 7, 93-110.

Emmison, M. (1988). On the interactional management of defeat. Sociology, 22, 233-251.

File, K. A. (2017). ‘I didn't know you were allowed two goalkeepers’: How football managers negotiate invitations to criticise referees in the media. In D. Caldwell et al. (Eds.), The discourse of sport: Analyses from social linguistics (pp. 71-91). New York, NY: Routledge.

Gallmeier, C. P. (1987). Putting on the game face: The staging of emotions in professional hockey. Sociology of Sport Journal, 4, 347-362.

Goodwin, M. H. (1983). Aggravated correction and disagreement in children's conversations. Journal of Pragmatics, 7, 657-677.

Goodwin, C., \& Goodwin, M. H. (1986). Gesture and coparticipation in the activity of searching for a word. Semiotica, 62, 51-75.

Goosby-Smith, J. (2009). NFL head coaches as sensegiving change agents. Team Performance Management: An International Journal, 15(3), 202-214.

Heinemann, T. (2009). Participation and exclusion in third party complaints. Journal of Pragmatics, 41, 2435-2451. 
Holt, R. (2000). The discourse ethics of sports print journalism. Journal of Culture, Sport, Society, 3, 88-103.

Hutchby, I. (1992). Confrontation talk: Aspects of ‘interruption’ in argument sequences on talk radio. Sociology, 3, 343-371.

Jefferson, G. (1974). Error correction as an interactional resource. Language in Society, 2,181199.

Kassing, J. W., \& Anderson, R. L. (2014). Contradicting coach or grumbling to teammates: Exploring dissent expression in the coach-athlete relationship. Communication \& Sport, 2, 172-185.

Kassing, J. W., Billings, A. C., Brown, R. S., Halone, K. K., Harrison, K., Krizek, B., . . . Turman, P. D. (2004). Communication in the community of sport: The process of enacting, (re)producing, consuming, and organizing sport. Annals of the International Communication Association, 28, 373-409.

Kurtz, J. B. (2019). With malice towards all? Moral authority, violence, and the (affective) discipline of basketball (bodies). Communication \& Sport, 7, 157-175.

Llewellyn, J. (2003). Coachtalk: Good reasons for winning and losing. In R. S. Brown \& D. O'Rourke (Eds.), Case studies in sport communication (pp. 141-158). Westport, CT: Praeger.

Mellick, M., Fleming, S., \& Davies, G. (2007). An interpretive analysis of interpersonal communication: a case study from elite rugby union match officiating. International Journal of Performance Analysis in Sport, 7, 92-105.

Monzoni, C. M. (2008). Introducing direct complaints through questions: The interactional achievement of 'pre-sequences'? Discourse Studies, 10, 73-87. 
Peräkylä, A., \& Ruusuvuori, J. (2012). Facial expression and interactional regulation of emotion. In A. Peräkylä, \& M.-L. Sorjonen (Eds.), Emotion in interaction (pp. 64-91). Oxford: Oxford University Press.

Pomerantz, A. M. (1984). Agreeing and disagreeing with assessments: Some features of preferred/dispreferred turn shapes. In J. M. Atkinson, \& J. Heritage (Eds.), Structures of social action: Studies in conversation analysis (pp. 57-101). Cambridge: Cambridge University Press.

Pomerantz, A. (1986). Extreme case formulations: A way of legitimizing claims. Human Studies, 9, 219-229.

Potter, J. (1996). Representing reality: Discourse, rhetoric and social construction. London: Sage.

Praschinger, A., Pomikal, C., \& Stieger, S. (2011). May I curse a referee? Swear words and consequences. Journal of Sports Science \& Medicine, 10, 341-345.

Rhys, C. (2016). Grammar and epistemic positioning in interviews: When assessment rules. Research on Language and Social Interaction, 49, 183-200.

Robinson, J. D. (2007). The role of numbers and statistics within conversation analysis, Communication Methods and Measures, 1, 65-75.

Ruusuvuori, J. \& Lindfors, P. (2009). Complaining about previous treatment in health care settings. Journal of Pragmatics, 41, 2415-2434.

Ruusuvuori, J., Asmuß, B., Henttonen, P, \& Ravaja, N. (2019). Complaining about others at work. Research on Language and Social Interaction, 52, 41-62.

Sanderson, J. (2009). "Thanks for fighting the good fight": Cultivating dissent on Blogmaverick.com. Southern Communication Journal, 74, 390-405.

Schegloff, E. (2005). On complainability. Social Problems, 52, 449-476.

Sidnell, J. (2010). Conversation analysis: An introduction. West Sussex: Wiley-Blackwell. 
Stokoe, E. (2009). Doing actions with identity categories: complaints and denials in neighbor disputes. Text \& Talk, 29, 75-97.

Traverso, V. (2009). The dilemmas of third-party complaints in conversation between friends. Journal of Pragmatics, 41, 2385-2399.

Waymer, D., \& Bradley, S. (2018). If professional athletes must talk with media, are they free to express how they truly feel? Examining celebrity-athlete emotional displays in media. Journal of Sports Media, 13, 1-25.

Wilton, A. (2017). The interactional construction of evaluation in post-match football interviews. In D. Caldwell et al. (Eds.), The discourse of sport: Analyses from social linguistics (pp. 72-112). New York, NY: Routledge. 